Voix et Images

voixetimages

\title{
Regards lucides sur la société
}

\section{Gaëtan Lévesque}

Volume 8, numéro 1, automne 1982

\section{Madeleine Gagnon}

URI : https://id.erudit.org/iderudit/200374ar

DOI : https://doi.org/10.7202/200374ar

Aller au sommaire du numéro

Éditeur(s)

Les Presses de l'Université du Québec

ISSN

0318-9201 (imprimé)

1705-933X (numérique)

Découvrir la revue

Citer cet article

Lévesque, G. (1982). Regards lucides sur la société. Voix et Images, 8(1),

154-158. https://doi.org/10.7202/200374ar d'utilisation que vous pouvez consulter en ligne.

https://apropos.erudit.org/fr/usagers/politique-dutilisation/ 


\title{
Regards lucides sur la société
}

\author{
par Gaëtan Lévesque, Université du Québec à Montréal
}

En consultant les quotidiens, on se rend compte que la violence est de plus en plus présente dans notre société, tout comme la drogue. On n'a qu'à se promener sur la rue Saint-Denis pour se faire offrir du "pot» ou du "hasch", beau, bon, pas cher, "for a good trip", et constater que la drogue est à la portée de tous. Ces phénomènes n'échappent pas à nos écrivains et il n'est pas rare de retrouver, chez nos romanciers et nos romancières, une image de cette réalité.

\section{Visions d'Anna}

«... on en voyait mourir souvent d'une overdose, à l'urgence, mais on ne pensait jamais à sa propre fille, seulement aux autres, c'était comme les homosexuels, on en disait parfois du bien, parfois du mal, mais on ne pensait jamais à Liliane, à sa propre fille..." (p. 45).

Après trois ans d'absence de notre petit milieu littéraire (son dernier roman, le Sourd dans la ville ${ }^{1}$, date de 1979) Marie-Claire Blais propose les visions que de jeunes adolescentes jettent sur notre société et qui briment leur vécu.

Les Visions $d^{\prime} A n n a^{2}$, qui sont aussi les visions de Michelle, la jeune droguée et Liliane, l'homosexuelle, les deux autres protagonistes de ce roman, présentent un monde qui court vers sa destruction et où l'on est dans l'impossibilité de communiquer.

Entre le « hasch» et la «mari», en passant par la mescaline, la cocaine et l'héroïne, ces jeunes adolescentes désabusées promènent leur ennui, leur difficulté de vivre et le goût de vivre leur sexualité librement, dans un monde d'adultes qui voudraient voir ces jeunes marginaux se soumettre à leur loi, la loi de la normalité : «l'ordre» d'une certaine petite bourgeoisie.

Raymonde, la mère d'Anna, séparée de son mari, travaille à l'Institut Correctionnel où il y a tant de jeunes délinquantes qu'on ne sait plus où les loger. Elle voit sa fille Anna qui vit les mêmes problèmes que ces jeunes et se sent impuissante devant ces faits, tout comme les parents de Michelle et 
Liliane, essaient de comprendre ces deux jeunes filles qui ont tout pour être heureuses et qui, malgré cela, réussissent à être malheureuses.

Tout au long du roman, la possibilité d'établir une relation entre les parents et les adolescentes s'avère des plus difficiles, mais à la fin, l'auteur laisse entrevoir une possibilité de communication entre Raymonde et sa fille Anna :

«... Anna ouvrait la porte de sa chambre, elle quittait son île, Raymonde venait près d'elle, sans oser le croire, elle pensait en serrant Anna contre son ccur, je pense que cette fois elle est de retour.» (p. 169).

Marie-Claire Blais décrit l'univers de la délinquance de façon très objective. Dès les premières pages, l'expérience de la romancière s'affirme et le lecteur prend plaisir à suivre le cheminement de ces jeunes à travers une écriture fascinante et particulière à cette écrivaine, qu'il est toujours agréable de lire.

\section{Les Faux-fuyants}

Il aura fallu trois ans aussi à Monique LaRue pour nous donner son deuxième roman, les Faux-fuyants ${ }^{3}$. Son premier roman, la Cohorte fictive ${ }^{4}$, publié en 1979, apparaît comme une sorte d'introduction à sa dernière publication. On y retrouve une continuité des personnages pris dans la problématique "vie-mort», fuite et désespoir.

On remarque, dans les faux-fuyants, la même thématique que chez Marie-Claire Blais, l'incommunicabilité des êtres entre eux et le problème de la drogue, auxquels s'ajoute la fuite des parents et des adolescents qui les conduit vers le désespoir. En fait, au contraire de M.-C. Blais, où la famille est présente tout en étant absente, chez Monique LaRue, c'est la famille éclatée sous toutes ses formes, l'absence totale.

Maurice et Zella ont mis au monde Klaus et Elodie, deux jumeaux dont la dernière est muette, débile mentale et vit sous la protection de son frère Klaus. Maurice, le père, refuse la paternité, s'enfuit du foyer et, en bon intellectuel universitaire, se consacre à ses travaux et à ses recherches tout en vivant son homosexualité, jusqu'au jour où une lettre de son fils lui rappelle qu'il a mis au monde des enfants et que lui, Klaus, ne lui pardonnera jamais cet acte insensé. C'est de nouveau le désespoir et la fuite vers l'Europe.

Zella, la mère, sombre dans l'alcool et fuit vers la Californie sur les conseils de sa psychiatre, qui est elle-même un peu débalancée. Zella baise avec les hommes, avec les femmes, essaie toutes les thérapies possibles, sans grand succès. Puis arrive la mort accidentelle (ou est-ce un suicide?) qui la délivrera de ce voyage impossible dans la «vie-mort».

Les jumeaux, principaux personnages de ce roman, sont deux jeunes de vingt ans quifument $n$ 'importe quoi, boivent de la bière et écoutent les Rolling 
Stones; on est loin du “Moi, j'bois mon lait comme ça m'plaît »... Inséparables depuis leur naissance, ils doivent se quitter lorsque Elodie part vivre à Sept-Îles avec un camionneur de qui elle attend un enfant. Klaus, qui perd la moitié de sa vie, son double en fait, se retrouve à Montréal, où il rencontre une ancienne amie de cégep, avec qui il partira "triper» vers la Floride; sans savoir à quel endroit précis, ni même pourquoi. Ce départ semble apporter une délivrance dans un ailleurs indéfinissable mais qui conduira peut-être Klaus vers une vie meilleure.

Ce roman suit le rythme des émotions, l'écriture se fait violente par moments, mais aussi toute douceur dans des passages pleins de tendresse. Les Faux-fuyants est aussi "flyé" que les jeunes des années 80 aux prises avec leur problème existentiel dans le voyage insupportable de la "vie-mort». Tout comme dans la Cohorte fictive, Monique LaRue analyse en profondeur ses personnages et donne un roman bien construit qui ne laisse pas le lecteur indifférent. Décrire la vie des jeunes adolescents qui ont de la difficulté à s'adapter à un monde qui ne leur apporte que désillusions n'est pas chose facile et Monique LaRue réussit ce tour de force remarquablement.

\section{Ah, l'amour l'amour}

Pour sa deuxième publication en "prose», Noël Audet a décidé de "faire du pouce" vers la mer, vers sa Gaspésie natale, vers l'amour. Ceux qui ont lu Quand la voile faseille ${ }^{5}$ se souviendront que la quatrième partie de ce livre s'intitulait: "Une simple histoire d'amour" et on pouvait prédire qu'il récidiverait. Et c'est ce qu'il fait dans Ah, l'amour l'amour'.

En faisant le tour de la péninsule gaspésienne, André, le narrateur, rencontre Astrid à Rivière-du-Loup et ils s'associent pour faire le voyage. Mais très vite le rythme cardiaque du jeune roméo s'accélère, il ne fait pas d'extra-systoles, mais tout juste. Et c'est la lente approche vers cette compagne qui se laisse séduire difficilement: «- Moi ma tente, toi ta tente, décréta Astrid...» (p. 17). Ce n'est qu'à Gaspé que les jeunes tourtereaux passent à l'initiation. Et le voyage continue jusqu'à Petite-Matane, pour se terminer en catastrophe une décennie plus tard.

Après le mariage, les enfants, le désir sexuel qui diminuait, Astrid remplace André par un dénommé Bruno et André refuse le triangle bien qu'il qualifie de "moderne» leur relation de couple... La mort accidentelle de leur fillette de six ans, Emmanuelle, amène la rupture définitive.

Parallèlement à cette histoire, où la narration fait des retours en arrière de dix ans et revient dans le présent, Liana s'installe dans la vie d'André, une nouvelle liaison commence. Liana, tout le contraire d'Astrid:

«Astrid ne savait pas recevoir et ça me faisait mourir. J'avais l'âme comme une fleur de bordel à force de proposer ma marchandise. Liana 
par contre a la passion où d'autres ont la peau: un effleurement léger et toute l'étreinte surgit, et l'on se retrouve en pleine communication des profondeurs. (p. 177).

Et Liana fait son nid chez André, «brin après brin». Le roman se ferme sur la mort du chat Chénier à qui André raconte ses malheurs comme son bonheur.

Audet est un spécialiste de l'écriture, ça se lit! II sait rendre ses personnages intéressants et sympathiques; il sait aussi étaler des sentiments sans que cela soit ennuyeux. Que ce soit avec Astrid ou Liana, les deux personnages féminins avec qui André vit des histoires d'amour très intenses, ou bien qu'il dialogue avec la mer ou son chat Chénier, on sent, chez l'auteur, le besoin d'exprimer, avec humour et tendresse, les sentiments du cœur amoureux. Cela nous donne un tour de la Gaspésie et des aventures amoureuses qu'il faut lire pour l'écriture mais aussi pour les talents de conteur de Noël Audet.

\section{Greenwich, ou la fuite vers le Sud}

II y a, dans la littérature québécoise, un thème qui n'est pas nouveau et que l'on retrouve régulièrement, la fuite vers le sud. Cette course à travers l'Amérique semble être un des seuls moyens dont disposent les héros de nos romans pour retrouver une identité qui leur échappe.

Chez Monique LaRue, dans les Faux-fuyants, Klaus fuit vers la Floride et Zella, sa mère, vers la Californie; chez Pierre Turgeon, le héros de la Première Personne ${ }^{7}$ part pour Los Angeles tenter de refaire sa vie et oublier celle laissée derrière lui. Par contre, le Voyageur distrait ${ }^{8}$ de Gilles Archambault va rencontrer une amie à Lowell dans le Massachusetts, pour écrire un essai sur l'œuvre de Jack Kerouac; prétexte qui lui servira à remettre en question sa vie à Montréal, ses sentiments pour l'être aimé en même temps que sa vision de l'écriture. Mais il n'y a pas seulement chez les "professionnels" de l'écriture que cette attirance vers le sud est une priorité; les jeunes auteurs ont aussi le goût d'exiler leur héros.

Michel Bélil a choisi Boston, la capitale du Massachusetts, pour son premier roman, Greenwich ${ }^{9}$. C'est un premier roman, mais non une première expérience d'écriture; il a déjà publié des nouvelles, le Mangeur de livres ${ }^{10}$ et Déménagement ${ }^{11}$.

Greenwich Lapeau, qui deviendra Poe à Boston, tente d'oublier les nombreux malheurs qu'il a vécus malgré ses vingt ans. La mort de ses parents et de son jeune frère, le suicide de deux de ses amis, l'avortement et la mort de son amie Calypse, le font fuir vers Boston à la recherche de la paix.

À la manière des personnages d'Edgar Allan Poe, Greenwich sera hanté par des souvenirs bizarres et macabres qui le suivront dans sa fuite contre 
la folie et Boston ne sera pas la ville par excellence pour le repos. Pendant son séjour, des millions de sauterelles envahissent la ville et l'état d'urgence est décrété jusqu'à la disparition inexplicable des sauterelles. Greenwich repartira alors vers le Mexique.

Michel Bélil écrit bien, la forme est excellente. Par contre, le contenu nous en fait voir de toutes les couleurs. Bien sûr, les thèmes traditionnels y sont bien représentés: les études, l'amour, la vie, la folie et finalement la mort. Mais on ne sait pourquoi l'auteur a intégré un élément fantastique (sauterelles) qui vient briser le déroulement de la lecture avec le résultat que le lecteur a de la difficulté à faire le lien entre cette partie fantastique et le début traditionnel du roman. On a l'impression d'être dans un autre univers romanesque. Il y a trop d'éléments qui nous font oublier la raison première de la fuite et c'est la trame du roman qui en souffre. Mais il s'agit ici d'un premier roman et c'est toujours celui-là que les auteurs s'empressent d'oublier; ainsi que le lecteur d'ailleurs!

1. Blais, Marie-Claire, Le Sourd dans la ville, (Prix du Gouverneur général), Montréal, Éd. Internationales Alain Stanké, 1979, 216 p.

2. Blais, Marie-Claire, Visions d'Anna, Montréal, Éd. Internationales Alain Stanké, 1982, $176 \mathrm{p}$.

3. LaRue, Monique, les Faux-fuyants, Montréal, Éd. Québec/Amérique, coll. «Littérature d'Amérique», 1982, 208 p.

4. LaRue, Monique, la Cohorte fictive, Montréal, Éd. L'Étincelle, 1979, 128 p.

5. Audet, Noël, Quand la voile faseille, Montréal, Éd. Hurtubise HMH, coll. l'Arbre, $1980,318 \mathrm{p}$.

6. Audet, Noël, Ah, l'amour l'amour, Montréal, Éd. Quinze, coll. Prose entière, 1981, $192 \mathrm{p}$.

7. Turgeon, Pierre, la Première Personne, Montréal, Éd. Quinze, coll. Prose entière, $1980,160 \mathrm{p}$.

8. Archambault, Gilles, le Voyageur distrait, (Prix David 1981), Montréal, Éd. Internationales Alain Stanké, 1981, $126 \mathrm{p.}$

9. Bélil, Michel, Greenwich, Montréal, Éd. Leméac, coll. Roman québécois, 1981, $232 \mathrm{p}$.

10. Bélil, Michel, le Mangeur de livres, Montréal, Éd. Cercle du livre de France/Pierre Tisseyre, 1979.

11. Bélil, Michel, Déménagement, Québec, Éd. Chasse-galerie, 1981, 80 p. 